\title{
Knowledge Attitude and Practice of Dengue Syndrome Management on the Basis of National Guideline by the Physicians of Dhaka City
}

\author{
Mohammad Rafiqul Islam¹, Sarmistha Biswas², Mohammad Robed Amin³, Md. Ridwanur Rahman ${ }^{4}$
}

\begin{abstract}
:
Objectives: Dengue is the fastest emerging arboviral infection. It was expected that the National Control Program will promote the use of the revised and updated version of the guideline for uniform clinical management of Dengue/DHF in the country with a view to reduce case fatality due to dengue. We tried to assess the knowledge attitude and practice of different tier of physicians on the basis of this guideline.

Method: Total 317 physicians from academic hospital, 57 physicians from non-academic hospital and 21 private practitioners took part in the survey.

Results: knowledge and attitude regarding management protocol of Dengue syndrome were significantly $(p<0.05)$ associated with different academic tier but practice was not significantly $(p<0.05)$ associated with different academics. The mean of accurate knowledge regarding management protocol of Dengue syndrome was significantly $(p<0.05)$ associated with training status but attitude and practice were not significantly $(p<0.05)$ associated with training status.

Conclusion: This study reveals that physicians possibly didn't went through the dengue guideline properly as their knowledge, attitude and practice were poor in each tier of professional practice. But dengue training improved knowledge regarding management of patients.
\end{abstract}

Key wards: Dengue, Dengue hemorrhagic fever (DHF)

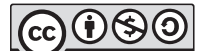

DOI: https://doi.org//0.3329/jom.v22il.5/386 Copyright: (C) 2021 Islam MR. This is an open access article published under the Creative Commons Attribution-NonCommercial-NoDerivatives 4.0 International License, which permits use, distribution and reproduction in any medium, provided the original work is properly cited, is not changed in any way and it is not used for commercial purposes.

Received: 20 November, 2020;

Accepted: 02 December, 2020

\section{Introduction:}

Dengue is the most rapidly spreading mosquito-borne viral disease in the world. In the last 50 years, incidence has increased 30-fold with increasing geographic expansion to

1. Dr. Mohammad Rafiqul Islam, Associate Professor of Medicine, Shaheed Suhrawardy Medical College.

2. Dr. Sarmistha Biswas, Associate Professor of Medicine, Dhaka Medical College.

3. Dr. Mohammad Robed Amin, Professor of Medicine, Dhaka Medical College

4. Prof. Md. Ridwanur Rahman, Professor \& Head, Department of Clinical Research, Universal Medical College Research Center.

Funded by Bangladesh Society of Medicine.

Corresponding author: Dr. Mohammad Rafiqul Islam, Associate Professor of Medicine, Shaheed Suhrawardy Medical College. Cell: 01753199796, E-mail: drrafiq73@yahoo.com new countries and, in the present decade, from urban to rural settings. Approximately 2.5 billion people live in dengue endemic countries ${ }^{1}$. According to $\mathrm{WHO}$, some 1.8 billion (more than $70 \%$ ) of the population at risk for dengue worldwide live in member states of the WHO South-East Asia Region and Western Pacific Region, which bear nearly $75 \%$ of the current global disease burden due to dengue. Historically, dengue has been reported predominantly among urban and peri-urban populations where high population density facilitates transmission. In Bangladesh, first documented case of dengue like fever occurred in 1964 popularly known as "Dacca fever" which later serologically proved as Dengue Fever ${ }^{2}$. Symptomatic dengue virus infections can present with a wide range of clinical manifestations, from a mild febrile illness to a life-threatening shock syndrome ${ }^{3,4}$. Both viral and host factors are thought to contribute to the manifestations of disease in each infected 
individual. The risk of severe disease is much higher in sequential rather than in primary dengue infection. The etiologic agent (DENV) belongs to the Flavi viridae family and to the Flavivirus genus, with 4 serotypes ${ }^{5}$. Dengue is the most rapidly spreading mosquito-borne viral disease in the world ${ }^{6}$. The disease control division of Directorate General of Health services felt necessity of developing national guideline in 2000 for clinical management of dengue by customizing the SEARO/WHO guidelines turned to the prevailing local situations for providing appropriate management for Dengue patients to mitigate the morbidity, to prevent regrettable mortality and to create awareness for appropriate prevention and control. The second edition of the National guideline for clinical management of Dengue syndrome was updated in 2009. The third edition, 2013 of the national guidelines on dengue syndrome was updated with the view to early diagnosis and effective management of Dengue. Despite all these facts, understanding dengue guideline properly by the physicians who work in the field, have some ambiguity. On this background we want to conduct a knowledge, attitude and practice-based survey of the physicians of Dhaka city about dengue syndrome management on the light of National guideline 2013 (Third Edition).

\section{Methodology:}

It was an observational survey type study. Study was conducted at Dhaka City in 2016. Study Population were Physicians of Dhaka city. Survey questionnaire was set up and pre tested. Respondent was chosen from different tier of physicians. First group were from three Academic hospital and total respondents were 317. Second group of 59 respondent physicians were from three non-academic hospitals (Both private and Government medical Hospital). Third group of 21 respondent physicians were from private practitioner not engaged with any hospital. Total respondents were 397. Total 30 questions were set where 23 questions were knowledge based, 4 questions were attitude based and 3 questions were practice based. Each question had multiple steams (2-4). Survey was done simultaneously in different location. Ethical clearance was taken from the ethical committee of Dhaka Medical College. Data analysis was done on the basis of different academic tier, postgraduation and status of receiving dengue training. Data analysis was done SPSS 20 manufactured by Chicago Illinois.

\section{Results:}

Overall performance of knowledge attitude and practice of Dengue management were poor among different tier of professionals. In response to question regarding warning sign, $5 \%, 5.1 \%$ and $0 \%$ physicians of academic, nonacademic and private practitioners respectively responded with correct answer (Table 1). Question regarding early confirmation and management of Dengue syndrome $10.1 \%$, $6.8 \%, 9.5 \%$ physicians of academic, non-academic and private practitioners respectively responded with correct answer (Table 2). We assessed association among accurate knowledge, attitude and practice regarding management protocol of Dengue syndrome with different academic tier. The mean of accurate knowledge and attitude regarding management protocol of Dengue syndrome were significantly $(\mathrm{p}<0.05)$ associated with different academic tier but practice was not significantly $(\mathrm{p}<0.05)$ associated with different academics (table III). Then we assessed association of accurate knowledge, attitude and practice regarding management protocol of Dengue syndrome with postgraduation status of physicians. The mean of accurate knowledge, attitude and practice regarding management protocol of Dengue syndrome were not significantly $(p>0.05)$ associated with postgraduation status of physicians (Table IV). Finally, we assessed association of accurate knowledge, attitude and practice regarding management protocol of Dengue syndrome with training status on dengue management. The mean of accurate knowledge regarding management protocol of Dengue syndrome was significantly $(\mathrm{p}<0.05)$ associated with training status but attitude and practice were not significantly $(\mathrm{p}<0.05)$ associated with training status (Table V).

Table I

Knowledge regarding Dengue fever with warning sings constitute according to academic record

\begin{tabular}{|c|c|c|c|c|c|c|c|c|c|}
\hline \multirow{2}{*}{$\begin{array}{l}\text { Dengue fever with } \\
\text { warning sings constitu }\end{array}$} & \multicolumn{2}{|c|}{ Academic $(\mathrm{n}=317)$} & \multicolumn{2}{|c|}{ Non academic $(\mathrm{n}=59)$} & \multicolumn{2}{|c|}{ Private $(n=21)$} & \multicolumn{2}{|c|}{ Total $(\mathrm{n}=397)$} & \multirow[t]{2}{*}{ P value } \\
\hline & te $n$ & $\%$ & $\mathrm{n}$ & $\%$ & $\mathrm{n}$ & $\%$ & $\mathrm{n}$ & $\%$ & \\
\hline No response & 11 & 3.5 & 0 & 0 & 0 & 0 & 11 & 2.8 & \\
\hline Correct & 16 & 5 & 3 & 5.1 & 0 & 0 & 19 & 4.8 & \\
\hline Partially correct & 45 & 14.2 & 5 & 8.5 & 1 & 4.8 & 51 & 12.8 & \\
\hline Wrong & 245 & 77.3 & 51 & 86.4 & 20 & 95.2 & 316 & 79.6 & \\
\hline
\end{tabular}




\section{Discussion:}

This study was performed to assess the knowledge, attitude and practice about the management of Dengue Syndrome on the basis of updated National Guideline $\left[3^{\text {rd }}\right.$ version, 2013] by the physicians of Dhaka city. It revealed that there is a significant lacking of all these three factors among the study population; who were the physicians treating the patients in public and private sectors. The average knowledge about the Dengue disease was markedly poor in all the three groups- the physicians working in academic hospitals, nonacademic hospitals and clinics and also the general practitioners. This finding is consistent with the study in Southern Taiwan but inconsistent with the study in Saudi Arabia. ${ }^{7,8}$ It is also less than the study done by Ibrar Rafique et al ${ }^{9}$; where the average Dengue knowledge score is $62 \%$ and Thaver et al where the average knowledge score is $57 \%$ .${ }^{10}$ The result is much less than that of Singapore where primary care physicians could choose at least 2 out of 3 preferred answers in more than $90 \%$ of cases. ${ }^{11}$

More than two thirds of the participants were not aware about the warning signs which is a bit less than the study done in Pakistan, where half of the participants failed to diagnose Dengue hemorrhagic fever (DHF). ${ }^{9}$ It establishes the fact that, to diagnose and treat Dengue cases effectively in an endemic zone like Bangladesh, continuing medical education, regular updating the national guideline and regular training of the health-care workers is a must. ${ }^{12,13}$

Most participants failed to mention the tests for early diagnosis of Dengue Syndrome like NS1 antigen as PCR is not easily available in our settings. The Dengue serology tests are not useful to diagnose the disease in febrile early phase. Other useful tools like full blood count, serial follow up of the cases are equally important. That's why physician's clinical decision taking and awareness about the appropriate timing of performing the diagnostic tests of Dengue is very much important to improve the existing situation. ${ }^{11,14}$

Overall, the mean accurate knowledge and attitude regarding management protocol of Dengue Syndrome were significantly associated with different academic tier, but practice was not significantly associated. This finding is consistent with the study done in Southern Taiwan ${ }^{7}$ but inconsistent with the study in Singapore ${ }^{11}$.

The mean accurate knowledge regarding the management protocol of the updated National Guideline of Dengue management [ $3^{\text {rd }}$ version, 2013] was significantly associated with the training status; which again emphasizes on the necessity of continuing training for all groups of health-care workers and it should be very much profession-tailored. ${ }^{7}$ The national guideline should be practiced in all tiers of health-care delivery system.
In terms of limitations, this study did not stratify the demographic variables of the study population. It only included the physicians of the Dhaka city, which is the most endemic zone for Dengue in Bangladesh. Moreover, it did not include other health-care professionals.

\section{Conclusion:}

This study reveals a significant lack of knowledge as well as attitude and practice in the field of Dengue management. There is also lacking in the implementation of the National Guideline of Dengue Syndrome in the practical field. The study also reveals that training improves the knowledge on Dengue management. So, this problem should be addressed on an urgent basis by conducting regular training courses, arranging continuing medical education and workshops as well.

\section{References:}

1. WHO. Dengue and Dengue Haemorrhagic Fever. Factsheet No 117. Revised May, 2008. Geneva. World Health Organization; 2008. Available from: http://www.who.int/ mediacentre/factsheets/fs117/en. [Last accessed on $2016 \mathrm{Jul}$ 2016].

2. Mobarak MR, Islam R, Bhuiyan AK M T, Akand N, Begum F. Evaluation of Dengue Fever in A Tertiary Care Children Hospital of Bangladesh. Northern International Medical College Journal.2017;9: 274-77

3. Dengue Fever (DF) in Pakistan. Fridous Jahan. Asia Pac Fam Med. 2011; 10(1): 1. Published online 2011 Feb 24. doi: $10.1186 / 1447-056 \mathrm{X}-10-1$

4. Mairuhu AT, Wagenaar J, Brandjes DP, van Gorp EC. Dengue: an arthropod-borne disease of global importance. Eur J Clin Microbiol Infect Dis 2004; 23:425-33.

5. Westaway EG, Brinton MA, Gaidamovich S, Horzinek MC, Igarashi A, Kaariainen L, et al. Flaviviridae. Intervirology. 1985;24:183-92

6. WHO (2009) Dengue: Guidelines for diagnosis, treatment, prevention andcontrol. WHO Press, World Health Organization and the Special Programme for Research and Training in Tropical Diseases, France. Available: http:// whqlibdoc.who.int/publications/2009/9789241547871_ eng.pdf. Accessed 11August 2012. (14) (PDF) The Effects of Weather and Climate Change on Dengue. Available from: https://www.researchgate.net/publication/258639686 The_Effects_of_Weather_and_Climate_Change_on Dengue [accessed May 02 2020].

7. Tzong- Shiann Ho, Mei- Chih-Huang, Shin-Min Wang, Hsiang Chou Hsu, Ching-Chuan Liu. Knowledge, attitude, and practice of dengue disease among healthcare professionals in Southern Taiwan. Journal of the Formosan Medical Association.2013;112:18-23. 
8. Abdullah Azaharani. Knowledge and practice of primary health-care physicians regarding the dengue fever in Makkah Al-Mokarramah city;2013. International Journal of Medical Science and Public Health.2015;4(2):266.

9. Ibrar Rafique, Muhammad Arif Nadeem Saqib, Shajee siddique et al. Dengue knowledge and its management practices among physicians of major cities of Pakistan.J Pak Med Assoc.2015;65(4):392-396.

10. Thaver AM, Sobani ZA, Qazi F, Khan F, Khan M, Zafar A, Beg MA. Assessing the need for training general practitioners' knowledge, attitude and practice concerning dengue and malaria in Karachi, Pakistan. Int health. 2011;3;126-30.

11. Linda K Lee, Tun Linn Thein, Changa Kurukularatne, Victor CH Gan, David C Lye, yee sin Leo. Dengue Knowledge,
Attitudes, and Practices among Primary Care Physicians in Singapore.. Ann Acad Med Singapore. 2011;40:533-8.

12. Bloom BS. Effects of continuing medical education on omproving physician clinical care and patient health: a review of systemic reviews. Int J Technol Assess Health Care. 2005;21:380-5.

13. Xu G, Veloski J, Hojat M, Politzer RM, Rabinowitz H, rattner SL.Factors influencing primary care physicians' choice to practice in medically undeserved areas.Acad Med.1997;72:5109-11.

14. Tanner I, Scheiber M, Low JG, Ong A et al. Decision tree algorithms predict the diagnosis and outcome of dengue fever in the early phase of illness.PLoS Negl Trop Dis. 2008;2:e 196. 